\title{
Stem cells with fused gene expression of cytosine deaminase and interferon- $\beta$ migrate to human gastric cancer cells and result in synergistic growth inhibition for potential therapeutic use
}

\author{
KYOUNG-YOON KIM ${ }^{1}$, BO-RIM YI ${ }^{1}$, HYE-RIM LEE $^{1}$, NAM-HEE KANG ${ }^{1}$, \\ EUI-BAE JEUNG ${ }^{1}$, SEUNG U. KIM ${ }^{2,3}$ and KYUNG-CHUL CHOI ${ }^{1}$ \\ ${ }^{1}$ Laboratory of Veterinary Biochemistry and Immunology, College of Veterinary Medicine, \\ Chungbuk National University, Cheongju, Chungbuk, Republic of Korea; ${ }^{2}$ Division of Neurology, \\ Department of Medicine, University of British Columbia, Vancouver, British Columbia, Canada; \\ ${ }^{3}$ Medical Research Institute, Chung-Ang University College of Medicine, Seoul, Republic of Korea
}

Received September 14, 2011; Accepted November 9, 2011

DOI: $10.3892 /$ ijo.2011.1288

\begin{abstract}
Genetically engineered stem cells (GESTECs) producing suicide enzymes and immunotherapeutic cytokines have therapeutic effects on tumors, and may possibly reduce the side effects of toxic drugs used for treatments. Suicide enzymes can convert non-toxic pro-drugs to toxic metabolites that can reduce tumor growth. Cytosine deaminase (CD) is a suicide enzyme that metabolizes a non-toxic pro-drug, 5-fluorocytosine (5-FC), into the cytotoxic agent, 5-fluorouracil (5-FU). As an immunotherapeutic agent, human interferon- $\beta$ (IFN- $\beta$ ) has anticancer effects. In this study, we used modified human neural stem cells (HB1.F3) expressing the Escherichia coli (E. coli) CD gene (HB1. F3.CD) or both the CD and human IFN- $\beta$ genes (HB1.F3.CD. IFN- $\beta$ ) and evaluated their effectiveness on gastric carcinoma cells (AGS); migration of GESTECs to AGS was analyzed as well as formation of 5-FU and IFN- $\beta$. Reverse transcriptionpolymerase chain reaction (RT-PCR) was used to confirm the expression of CD and IFN- $\beta$ genes in GESTECs along with confirming the production of chemoattractant molecules such as stem cell factor (SCF), CXCR4, c-Kit, vascular endothelial growth factor (VEGF) and VEGF receptor 2 (VEGFR2). In addition, by co-culturing GESTECs with AGS in the presence of 5-FC, we were able to confirm that cancer growth was inhibited, along with a synergistic effect when the CD and
\end{abstract}

Correspondence to: Dr Kyung-Chul Choi, Laboratory of Veterinary Biochemistry and Immunology, College of Veterinary Medicine, Chungbuk National University, Cheongju, Chungbuk 361-763, Republic of Korea

E-mail: kchoi@cbu.ac.kr

Key words: gastric cancer, therapeutic stem cells, cytosine deaminase, interferon- $\beta$
IFN- $\beta$ genes (HB1.F3.CD.IFN- $\beta$ ) were co-expressed. Indeed a marked anticancer effect was demonstrated when the $\mathrm{CD}$ and IFN- $\beta$ genes were expressed together compared to expression of the CD gene alone (HB1.F3.CD). According to a modified transwell migration assay, the migration of GESTECs toward AGS was confirmed. In conclusion, these data suggest potential application of GESTECs to gastric cancer therapy, due to a remarkable synergistic effect of $\mathrm{CD}$ and IFN- $\beta$ genes in the presence of 5-FC. Additionally, the tumor-selective migration capability in vitro suggests that GESTECs are a potential anticancer therapy candidate that may result in minimal side effects compared to the conventional chemotherapy.

\section{Introduction}

Gastric adenocarcinoma is major type of the gastric cancer (1) and in most cases, they are detected in advanced stages. Despite the development of various treatments (e.g., gastrectomy, chemotherapy, radiation therapy) gastric adenocarcinoma ranks second in deaths caused by cancer, although the incidence rate ranks fourth (2). Several drugs are being used in gastric cancer therapy including 5-fluorouracil (5-FU) or its analog capecitabine, BCNU (carmustine) as well as methylCCNU (semustine), doxorubicin (Adriamycin), mitomycin C, cisplatin and Taxotere $(3,4)$. Although chemotherapy has been used for a long time, there is no clear standard of care and since gastric cancers are not particularly sensitive to these drugs, chemotherapy is mostly used to reduce the size of the tumor before surgery or used as adjuvant therapy (5). Since the selectivity of the drugs is low, treatments typically include systemic toxicity (3). To reduce these side effects, further studies are needed for safer and efficient treatment for gastric cancer (5-7).

Stem cells have recently become of great interest for researchers with the possibility of clinical use in cancer treatment. While traditional chemotherapy involves administration of manufactured drugs, genetically engineered stem 
cells (GESTECs) induces cells to produce the therapeutic agent $(8,9)$. This technique enables one to replace damaged genes or insert additional genes with a new function. For example, human neural stem cells (hNSCs) are one of the candidate stem cells showing therapeutic potential and tumor tropism for the treatment of malignant tumors in the human brain including medulloblastomas and gliomas (10-12). This supports the possibility of using hNSCs as a gene carrier to the tumor site as well as a tumor-specific enzyme/pro-drug system with concomitant prodrug administration (13). HB1.F3 is an immortalized hNSC derived from human fetal brain at 15 weeks of gestation by an amphotropic, replication-incompetent retroviral vector v-myc $(14,15)$. Clonal HB1.F3.CD cells were derived from parental HB1.F3 cells transfected with an Escherichia coli (E. coli) cytosine deaminase (CD) gene (14). Additionally, clonal HB1.F3.CD.IFN- $\beta$ cells were derived from parental HB1.F3.CD cells and their cells express both E. coli $\mathrm{CD}$ and human interferon- $\beta$ (IFN- $\beta$ ) genes (8). This clonally isolated, multi-potent $\mathrm{hNSC}$ has the ability to self-renew and to differentiate into cells of neuronal and glial lineages both in vivo and in vitro (14).

The CD/5-fluorocytosine (5-FC) system is a gene-directed enzyme/pro-drugs therapy (GEPT) (16-20) which converts the non-toxic prodrug 5-FC into the cytotoxic metabolite, 5-FU $(21,22)$. 5-FU inhibits DNA synthesis in cells and results in cytotoxicity $(23,24)$. This CD/5-FC GEPT system has been tested experimentally against several types of tumors including colorectal and prostate cancers (25-27).

In this study, we investigated the synergistic effect of IFN- $\beta$ with the CD/5-FC GEPT system. The proinflammatory cytokine, IFN- $\beta$ demonstrated antitumor activity by suppressing angiogenesis, tumor growth and metastasis $(28,29)$. The use of this pro-drug seems to be less toxic compared to using active anticancer drugs, but there is a difficulty in delivering the converting enzymes to the exact tumor site for selective activity. To reduce the side effect of therapeutic drugs and increase their effect, many researchers are focusing on genetargeting therapy that selectively works on cancer cells $(30,31)$. Therefore, we investigated whether the synergistic effect of the two systems can increase the efficiency of the treatment for gastric cancer.

Its therapeutic capacity in brain tumors as well as its tumortropic properties and migratory abilities makes GESTECs a potential candidate for invasive tumors (10-12,32). By delivering genes to selective tumor cells, GESTECs expressing fusion genes (i.e., CD and IFN- $\beta$ ) may have a synergic antitumor effect on gastric cancer cells.

\section{Materials and methods}

Cell culture. AGS, a human gastric adenocarcinoma cancer cell was originally derived from fragments of a tumor from a patient (Korean Cell Line Bank, Seoul, Korea). The cells were cultured in RPMI (PAA Laboratories GmbH, Linz, Austria) supplemented with $10 \%$ (v/v) fetal bovine serum (FBS; Hyclone Laboratories, Inc., Logan, UT, USA), 1\% HEPES (Invitrogen Life Technologies, Carlsbad, CA, USA), 1\% penicillin/streptomycin (Cellgro Mediatech, Inc., Manassan, VA, USA) and $0.1 \%$ antimycoplasmal plasmocin (Invivogen, San Diego, CA, USA) at $37^{\circ} \mathrm{C}$ in a humidified atmosphere of $5 \% \mathrm{CO}_{2}-95 \%$ air. $\mathrm{HB} 1$.
F3, HB1.F3.CD, HB1.F3.CD.IFN- $\beta$ (Chungang Universuty, Seoul, Korea) and the bovine fibroblast (Bovine FB) cells (Chungbuk National University, Cheongju, Korea) were cultured in DMEM (Hyclone Laboratories, Inc.) supplemented with $10 \%$ FBS, $1 \%$ penicillin $\mathrm{G}$ and streptomycin, $1 \%$ HEPES and $0.1 \%$ plasmocin at $37^{\circ} \mathrm{C}$ in a humidified atmosphere of $5 \%$ $\mathrm{CO}_{2}-95 \%$ air. Cells were trypsinized with $0.05 \%$ trypsin/ $0.02 \%$ EDTA (PAA Laboratories) in $\mathrm{Mg}^{2+} / \mathrm{Ca}^{2+}$-free HBSS.

Reverse-transcription polymerase chain reaction (RT-PCR). According to recent findings, the tumor tropism of the hNSCs are mediated by several chemoattractants and interaction with their specific receptors including stem cell factor $(\mathrm{SCF}) / \mathrm{c}-\mathrm{Kit}$ (33), stromal cell-derived factor 1 (SDF-1)/CXC chemokine receptor 4 (CXCR4) (34) and vascular endothelial growth factor (VEGF)/VEGF receptors VEGFR1 and VEGFR2 (32). The presence of these chemoattractants and related receptors in AGS were detected by RT-PCR.

Extraction of RNA was performed using the TRIzol reagent (Invitrogen Life Technologies). Using random primers, singlestranded cDNA was synthesized from $1 \mu \mathrm{g}$ of total RNA by M-MLV RT (iNtRON Biotechnology, Sungnam, Kyeonggido, Korea). The prepared cDNA from this procedure was used in the following PCR reactions performed with $0.2 \mu \mathrm{mol} / 1$ of each sense and antisense primers, 2.5 units of Taq polymerase (iNtRON Biotechnology), $0.2 \mathrm{mmol} / 1$ deoxynucleotide mix (iNtRON Biotechnology) and 10X PCR buffer (iNtRON Biotechnology). PCR for these chemoattractant factors (ligands and receptors) and glyceraldehyde-3-phosphate dehydrogenase (GAPDH) as a positive control was carried out for 30 cycles using PTC-100 (MJ Research, Inc., Waltham, MA, USA). PCR cycles were composed of a denaturation reaction at $95^{\circ} \mathrm{C}$ for $30 \mathrm{sec}$, annealing reaction at $58^{\circ} \mathrm{C}$ for $30 \mathrm{sec}$ and extension reaction at $72^{\circ} \mathrm{C}$ for $30 \mathrm{sec}$. The results were analyzed on a $1.5 \%$ agarose gel containing ethidium bromide (EtBr). The sense and antisense primers and the predicted sizes of the RT-PCR reaction products are shown in Table I.

Cell growth assay. To investigate the effect of 5-FC and 5-FU in gastric adenocarcinoma cells (4,000 cells/well), AGS were seeded in 96-well plates and cultured in $0.1 \mathrm{ml}$ medium with 5\% FBS. After a 24-h pre-incubation, HB1.F3, HB1.F3.CD, and HB1.F3.CD.IFN- $\beta$ cells were added to the cultures in medium containing 5\% FBS and incubated for $24 \mathrm{~h}$ before treatment with 5-FC or 5-FU. On the day of treatment, 5-FC and 5-FU (Sigma-Aldrich Corp., St. Louis, MO, USA) were serially diluted with phosphate-buffered saline (PBS; final concentration 100, 200,300, 400 and $500 \mu \mathrm{g} / \mathrm{ml})$ and the cells were treated for 4 days. An MTT [3-(4,5-dimethyl-thiazol2-yl)-2,5-diphenyltetrazolium bromide] assay was performed to measure cell viability on Day 7. MTT solution (10 $\mu \mathrm{l}$ of stock at $5 \mathrm{mg} / \mathrm{ml}$ ) was added to each well in the plates and incubated for $4 \mathrm{~h}$ at $37^{\circ} \mathrm{C}$. Supernatants were removed and $100 \mu \mathrm{l}$ of dimethyl sulfoxide (DMSO, 99.0\%; Junsei Chemical Co., Ltd., Tokyo, Japan) was added to each well to dissolve the resultant formazan crystals. Optical densities were measured at $540 \mathrm{~nm}$ using an ELISA reader (VersaMan, Molecular Devices, CA, USA). An MTT assay was carried out in duplicate.

To investigate the difference of cell growth and the changes in the ratio of cancer cells to GESTECs, AGS (4,000 cells/ 
Table I. The oligonucleotide sequences of the primers used in this study and the predicted sizes of the PCR products.

\begin{tabular}{|c|c|c|c|}
\hline \multirow{3}{*}{$\frac{\mathrm{mRNA}}{\mathrm{CD}}$} & \multicolumn{2}{|r|}{ Oligo-sequences (5'-3') } & \multirow{2}{*}{$\frac{\text { Expected size (bp) }}{559}$} \\
\hline & Forward & GCGCGAGTCACCGCCAGCCACACCACGGC & \\
\hline & Reverse & GTTTGTATTCGATGGCTTCTGGCTGC & \\
\hline \multirow[t]{2}{*}{$\mathrm{SCF}$} & Forward & ACTTGGATTCTCACTTGCATTT & 505 \\
\hline & Reverse & CTTTCTCAGGACTTAATGTTGAAG & \\
\hline \multirow[t]{2}{*}{ c-Kit } & Forward & GCCCACAATAGATTGGTATTT & 570 \\
\hline & Reverse & AGCATCTTTACAGCGACAGTC & \\
\hline \multirow[t]{2}{*}{ CXCR4 } & Forward & СТСТCCAAAGGAAAGCGCAGGTGGACAT & 558 \\
\hline & Reverse & AGACTGTACACTGTAGGTGCTGAAATCA & \\
\hline \multirow[t]{2}{*}{ IFN- $\beta$} & Forward & AAAGAAGCAGCAATTTTCAG & 296 \\
\hline & Reverse & TTTCTCCAGTTTTTCTTCCA & \\
\hline \multirow[t]{2}{*}{ VEGF } & Forward & AAGCCATCCTGTGTGCCCCTGATG & 377 \\
\hline & Reverse & GCTCCTTCCTCCTGCCCGGCTCAC & \\
\hline \multirow[t]{2}{*}{ VEGFR2 } & Forward & ACGCTGACATGTACGGTCTAT & 438 \\
\hline & Reverse & GCCAAGCTTGTACCATGTGAG & \\
\hline \multirow[t]{2}{*}{ GAPDH } & Forward & ATGTTCGTCATGGGTGTGAACCA & 351 \\
\hline & Reverse & TGGCAGGTTTTTCTAGACGGCAG & \\
\hline
\end{tabular}

well) were seeded in 96-well plates and cultured in $0.1 \mathrm{ml}$ medium with $5 \%$ FBS. After a 24 -h pre-incubation, HB1.F3, HB1.F3.CD or and HB1.F3.CD.IFN- $\beta$ cells were added to the cultures in medium containing $5 \%$ FBS separately at $8.0 \times 10^{3}$, $1.6 \times 10^{4}$ and $2.4 \times 10^{4}$ cells/well and incubated for $24 \mathrm{~h}$ before treatment with 5-FC (Sigma-Aldrich Corp.). On the day of treatment, cells were treated with 5-FC (final concentration $500 \mu \mathrm{g} / \mathrm{ml}$ ) for 4 days. MTT assay was performed to measure cell viability on Day 7. MTT solution $(10 \mu \mathrm{l})$ was added to each well in the plates and they were incubated for $4 \mathrm{~h}$ at $37^{\circ} \mathrm{C}$. Supernatants were removed and $100 \mu 1$ of DMSO (Junsei Chemical Co., Ltd.) was added to each well to dissolve the resultant formazan crystals. Optical densities were measured at $540 \mathrm{~nm}$ using an ELISA reader (VersaMan, Molecular Devices). The MTT assay was carried out in duplicate.

In vitro migration assay. To investigate whether GESTECs are capable of migrating to gastric cancer cells, AGS and bovine FB ( $1 \times 10^{5}$ cells/well) were plated in 24 -well plates and incubated in RPMI and DMEM contained 10\% FBS for $6 \mathrm{~h}$ at $37^{\circ} \mathrm{C}$, respectively. The cells were then incubated with new serum-free media and incubated for $24 \mathrm{~h}$. Transwell plates ( $8 \mu \mathrm{m}$; BD Biosciences, Franklin Lakes, NJ, USA) coated with fibronectin $(250 \mu \mathrm{g} / \mathrm{ml}$; Sigma-Aldrich Corp.) were placed in the 24-well plates and incubated overnight. Using a general protocol, $2 \mu \mathrm{M}$ of chloromethylbenzamido-1,1'-dioctadecyl-3,3,3'-tetramethyl-indocarbocyanine perchlorate (CM-DiI; Invitrogen Life Technologies) was used to label the HB1.F3, HB1.F3.CD or HB1.F3.CD.IFN- $\beta$ cells $\left(1 \times 10^{5}\right.$ cells/well) that were plated in the upper chambers of the transwell plates and cultured in serum-free medium for $24 \mathrm{~h}$ at $37^{\circ} \mathrm{C}$. The next day, AGS and bovine FB were stained by addition of a 200-ng/ml 4',6-diamidino-2-phenylindole solution (DAPI; Invitrogen, Lift Technologies) and the plate was incubated for $10 \mathrm{~min}$ at $37^{\circ} \mathrm{C}$. Each well was washed with PBS and the upper side of the transwell membrane was

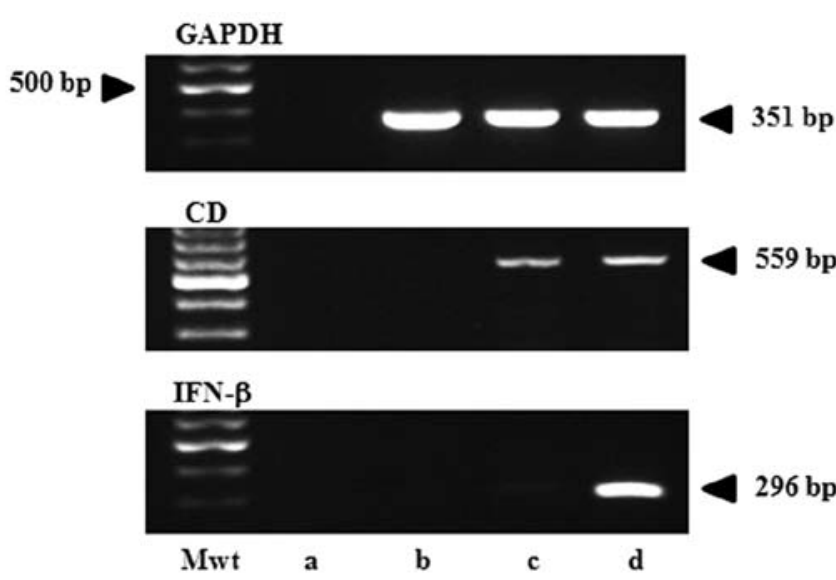

Figure 1. Expression of CD and IFN- $\beta$ genes in GESTECs. Expected products of $E$. coli $\mathrm{CD}$ or human IFN- $\beta$ genes in HB1.F3.CD and HB1.F3.CD.IFN- $\beta$ are shown at 559 and $296 \mathrm{bp}$, respectively. The cDNAs were synthesized from the mRNAs of HB1.F3, HB1.F3.CD and HB1.F3.CD.IFN- $\beta$ by RT and amplified by PCR. Then, these PCR products were confirmed by agarose gel electrophoresis. GAPDH was used as a control. Mwt, molecular weight marker; a, negative control without template; b, HB1.F3; c, HB1.F3.CD; d; HB1.F3.CD.IFN- $\beta$.

then scraped to remove cells that had not migrated into the membrane. Cells stained with CM-DiI and DAPI were examined by fluorescence microscopy (IX71 Inverted Microscope, Olympus, Japan).

Statistical analysis. The results of all cell growth assay experiments are presented as means \pm SD. One-way ANOVA was performed and a $\mathrm{P}<0.05$ was considered statistically significant.

\section{Results}

Confirmation of $C D$ and IFN- $\beta$ gene expression in GESTECs. The expression of CD and IFN- $\beta$ genes in HB1.F3, HB1.F3.CD 
A

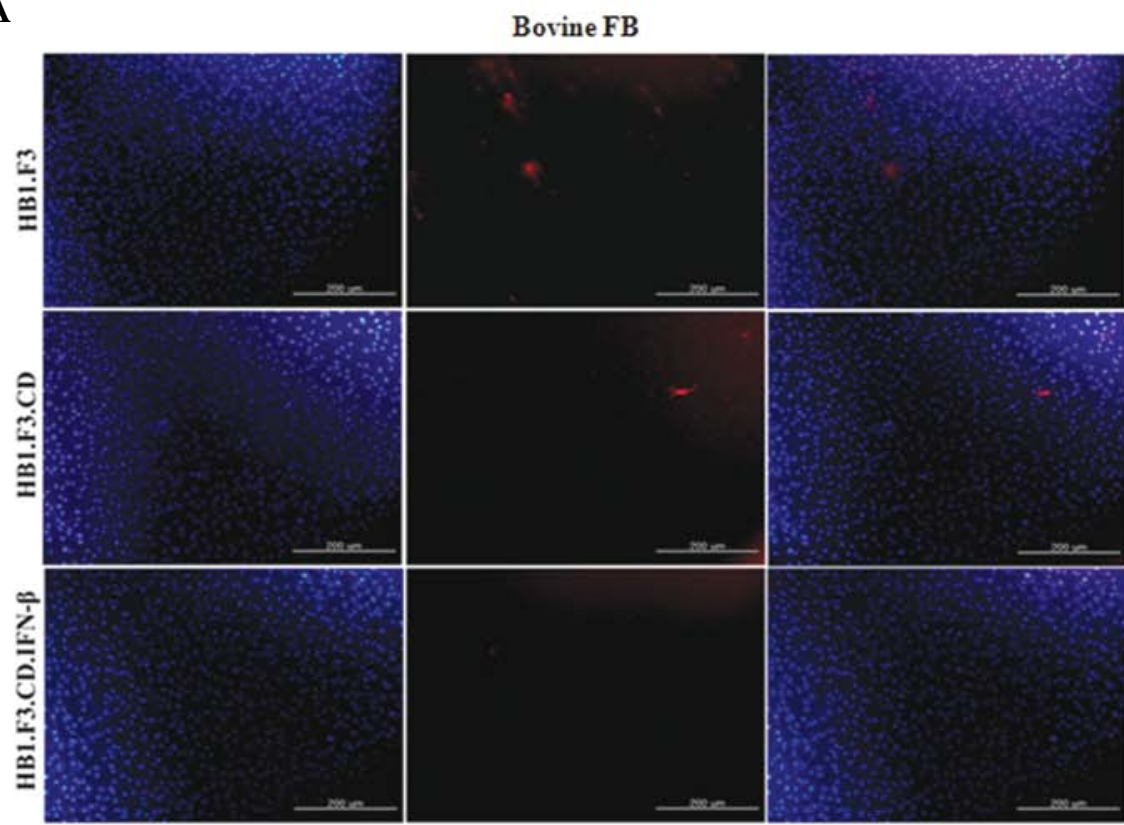

B

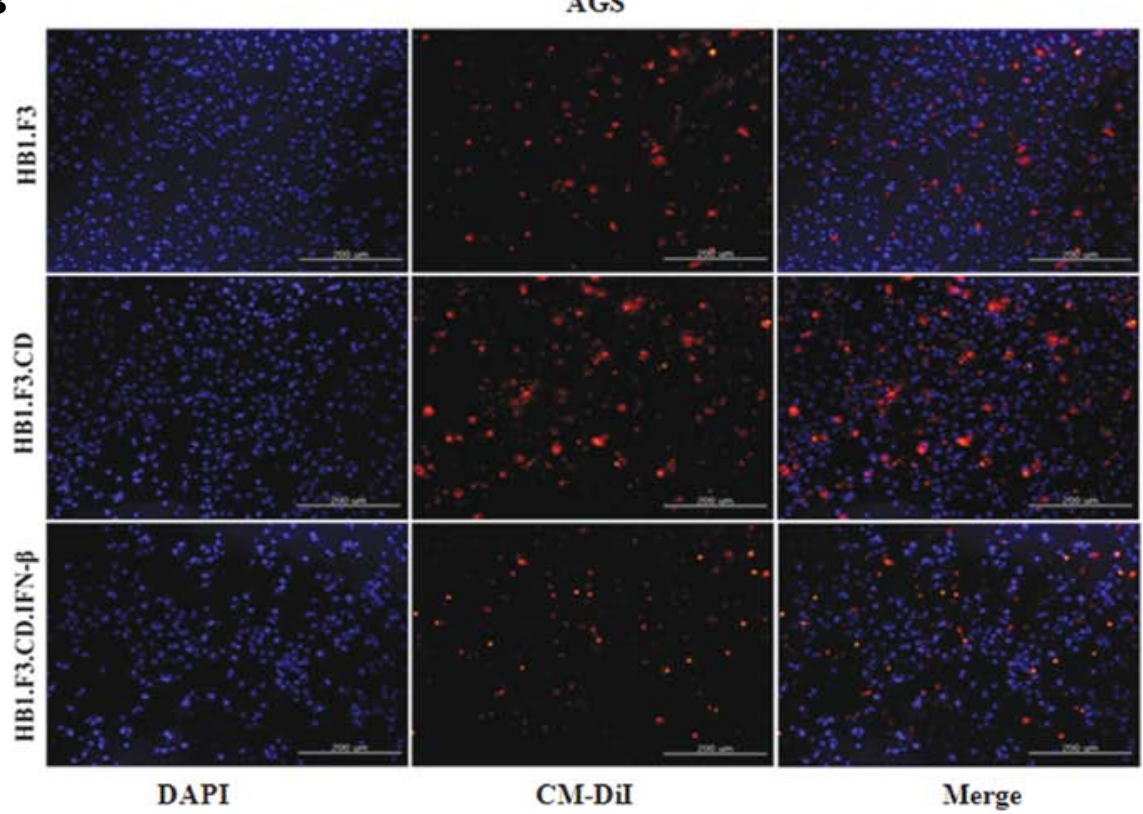

Figure 2. In vitro migration of GESTECs toward gastric cancer cells. The migratory capacity was assessed using a modified transwell migration assay. Twenty-four-well plates were pre-coated with fibronectin and HB1.F3, HB1.F3.CD or HB1.F3.CD.IFN- $\beta$ cells were added to the upper chamber of the insert $\left(1.0 \times 10^{5}\right.$ cells/well, red stained) after placing the transwell chamber above AGS and bovine FB cells. (A) Bovine FB (1.0x10 cells/well, blue stained) seeded in the chamber. (B) AGS seeded in the lower chamber (1.0x $10^{5}$ cells/well, blue stained). The inserts were collected and stained as previously described. The numbers of cells migrating into the membrane were counted using fluorescence microscopy (x100).

and HB1.F3.CD.IFN- $\beta$ cells were confirmed by RT-PCR. mRNA of the CD gene (559 bp) was confirmed in both HB1. F3.CD and HB1.F3.CD.IFN- $\beta$ cells demonstrating CD gene expression in HB1.F3.CD and HB1.F3.CD.IFN- $\beta$ cells (Fig. 1). In addition, the IFN- $\beta$ gene (296 bp) was expressed in HB1. F3.CD.IFN- $\beta$ cells, but not in HB1.F3 and HB1.F3.CD cells (Fig. 1). GAPDH was used as positive control and found based on the presence of its $351 \mathrm{bp}$ cDNA. Results of RT-PCR were confirmed by $1.5 \%$ agarose gel electrophoresis.

In vitro cell migration assay. To verify the migration capability of GESTECs toward the AGS, a modified transwell migration assay was performed. Using fluorescence microscopy, changes in CM-DiI stained hNSCs, HB1.F3, HB1.F3.CD and HB1. F3.CD.IFN- $\beta$ cells was performed. Compared with DAPIstained bovine FB as a control, AGS significantly increased cell migration of the GESTECs (Fig. 2).

Confirmation of chemoattractant ligands and receptors. To examine whether gastric cancer cells express chemoattractant factors, RT-PCR for several chemoattractant ligands and their related receptors were done in AGS. Results in Fig. 3 show the expression of SCF, CXCR4 and VEGF genes, but c-Kit and VEGFR2 were not expressed. According to these findings, it 

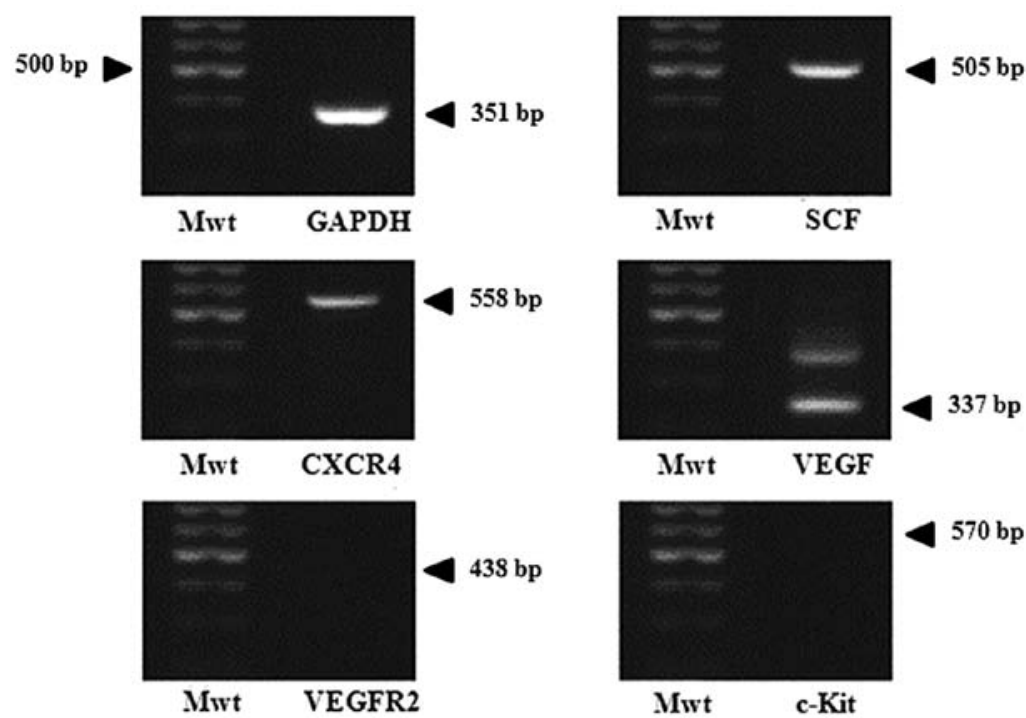

Mwt VEGF

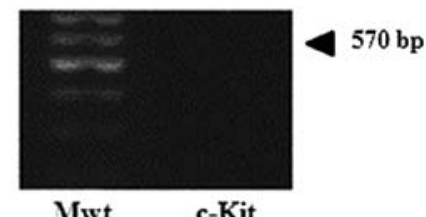

Figure 3. Expression of potential chemoattractant factors involved in tumor tropism and cell growth. The PCR products of GAPDH, SCF, CXCR4, VEGF, VEGFR2 and c-Kit were obtained by RT-PCR as described in Materials and methods. After cDNA synthesis, products were subjected to $1.5 \%$ agarose gel electrophoresis. GAPDH was employed as a positive control.

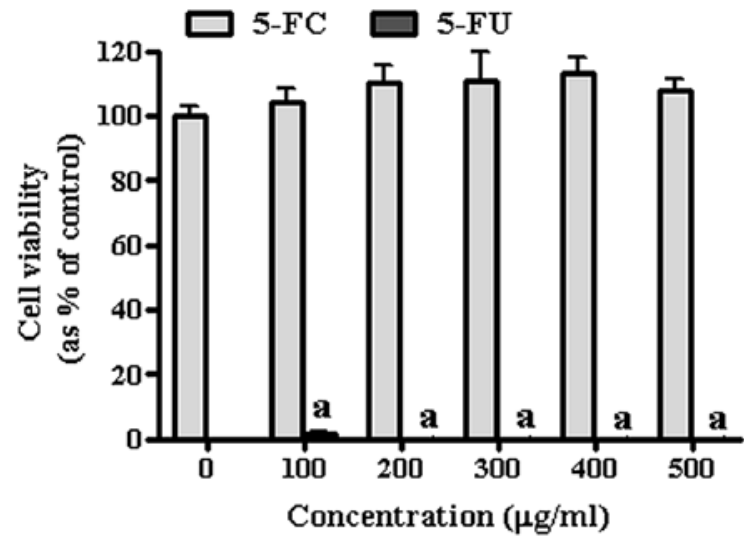

Figure 4. Effect of 5-FC and 5-FU on gastric cancer cell proliferation. Growth of gastric cancer cells were measured following treatments with increasing concentrations of 5-FC or 5-FU (relative fold-change compared to a control). AGS cells $\left(4.0 \times 10^{3}\right.$ cells/well) were seeded in 96 -well plates and were treated with either 5-FC or 5-FU at increasing concentrations $(100$, $200,300,400$ and $500 \mu \mathrm{g} / \mathrm{ml})$. Values represent the means $\pm \mathrm{SD}$ for three independent experiments. a, $\mathrm{P}<0.05$ vs. 5-FC.

can be assumed that AGS produces chemoattractant molecules and related receptors which induce migration of GESTECs.

Effect of 5-FC/5-FU on gastric cancer cells and GESTECs. To confirm the anticancer effect of HB1.F3, HB1.F3.CD and HB1.F3.CD.IFN- $\beta$ cells, cell viability assay was conducted using a co-culture system and confirmed by MTT assay. Prior to the co-culture experiment with of GESTECs, the effect of the prodrug 5-FC and its active metabolite 5-FU on AGS are shown in Fig. 4. According to these results, 5-FC did not appear to effect the growth of the gastric cancer cells. On the other hand, the growth inhibition effect of 5-FU was significant indicating AGS is highly sensitive to 5-FU, even at low concentration (100 $\mu \mathrm{g} / \mathrm{ml})$ (Fig. 4). To specifically determine the prodrug conversion efficiency of GESTECs, AGS were

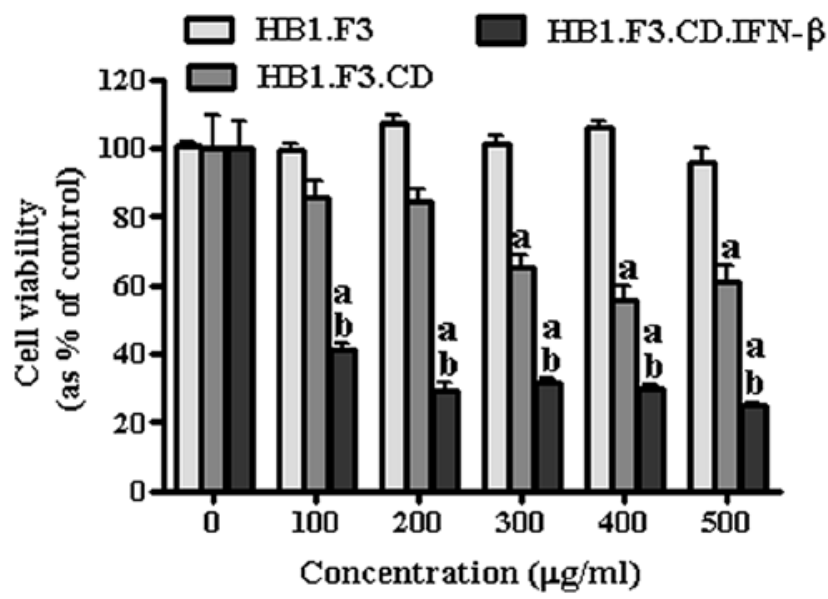

Figure 5. Effect of GESTECs and 5-FC on gastric cancer cell growth Proliferation of gastric cancer cells were measured following co-culture with GESTECs in the presence of increasing concentrations of 5-FC. AGS cells were seeded in 96-well plates and HB1.F3, HB1.F3.CD or HB1.F3.CD.IFN- $\beta$ cells $\left(8.0 \times 10^{3}\right.$ cells/well) were separately co-cultured with AGS, followed by treatment with 5-FC at increasing concentrations $(100,200,300,400$ and $500 \mu \mathrm{g} / \mathrm{ml})$. Values represent the means \pm SD for three independent experiments. a, $\mathrm{P}<0.05$ vs. HB1.F3; b, $\mathrm{P}<0.05$ vs. HB1.F3.CD.

co-cultured with each stem cell treated by 5-FC at different concentrations $(100,200,300,400$ and $500 \mu \mathrm{g} / \mathrm{ml})$ (Fig. 5) and cell viability was measured. HB1.F3 cells, the non-modified control NSC appeared not to inhibit cell growth at any concentration, while HB1.F3.CD cells started to inhibit cancer cell growth with 5 -FC treatment reached $300 \mu \mathrm{g} / \mathrm{ml}$. Impressively, HB1.F3.CD.IFN- $\beta$ cells showed significant inhibition at the lowest 5 -FC concentration $(100 \mu \mathrm{g} / \mathrm{ml})$. In the presence of the GESTECs, treatment of the 5-FC prodrug dose-dependently inhibited cancer cell growth in HB1.F3.CD and HB1.F3.CD. IFN- $\beta$ cells. 


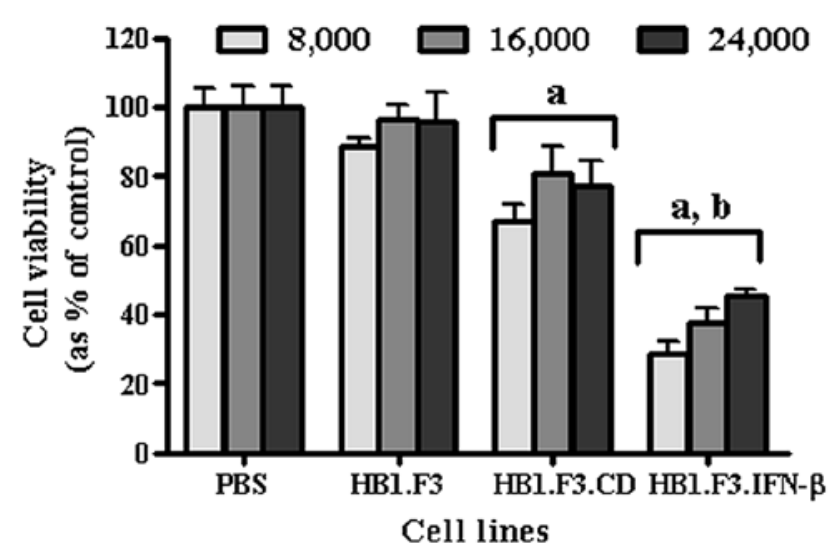

Figure 6. Effect of GESTEC cell numbers on gastric cancer cell proliferation. Proliferative levels of gastric cancer cells were examined following co-culture with increasing numbers of GESTECs in the presence of 5-FC. AGS cells $\left(4.0 \times 10^{3}\right.$ cells/well) were seeded in 96-well plates, and HB1.F3, HB1.F3.CD and HB1.F3.CD.IFN- $\beta$ cells were co-cultured with increasing cell numbers $\left(8.0 \times 10^{3}, 1.6 \times 10^{4}\right.$ and $2.4 \times 10^{4}$ cells/well). Values represent the means \pm SD for three independent experiments. $\mathrm{a}, \mathrm{P}<0.05$ vs. HB1.F3; b, $\mathrm{P}<0.05$ vs. HB1.F3.CD.

Furthermore, to verify whether the number of the stem cells affected the intensity of anticancer effect in gastric cancer cells, AGS $\left(4.0 \times 10^{3}\right.$ cells/well) were treated with 5-FC after co-culturing with different amounts of HB1.F3, HB1. F3.CD and HB1.F3.CD.IFN- $\beta$ cells $\left(8.0 \times 10^{3}, 1.6 \times 10^{4}\right.$ and $2.4 \times 10^{4}$ cells/well) (Fig. 6). After 5-FC treatment, cell viability was decreased in cells cultured with HB1.F3.CD and HB1. F3.CD.IFN- $\beta$ cells. Consistent with previous experiments, cancer cell viability was significantly decreased when $C D$ and IFN- $\beta$ genes were expressed together (HB1.F3.CD.IFN- $\beta$ ).

\section{Discussion}

This study is based on the theory that immortalized GESTECs have potential for gene therapy and cell replacement enabling treatment of neural disease and damage (14,35-40). Among several GESTECs, the NSCs are able to migrate to brain tumor sites and affect tumor growth both in vitro and in vivo $(11,12)$. In previous studies, using animal models, it was shown that when tumor cells were treated with HB1.F3.CD cells expressing the E. coli $\mathrm{CD}$ gene and systemic 5-FC administration together, the size of tumor cells were reduced $(30,31)$. At the same time when the tumor was treated only with HB1. F3.CD cells or 5-FC separately, there was no tumor cytotoxicity (12). Additionally, a recent study confirmed that human IFN- $\beta$ expressing GESTEC, that is HB1.F3.CD.IFN- $\beta$ cells, showed an anticancer effect compared to HB1.F3 cells (41).

The therapeutic capability of CD gene/5-FC modified GEPT system has been tested in several types of tumors including breast, prostate and colon $(20,25,42)$. The anticancer application of GESTECs is not well investigated in many other cancer cells. Therefore, in this study, we investigated the effect of CD/CD plus IFN- $\beta$ gene-expressing GESTECs in gastric cancer cells.

First, we tested the direct cytotoxicity of the CD gene/5-FC modified GEPT system with human IFN- $\beta$-expressing GESTECs. 5-FU, an inhibitor of thymidylate synthetase (43), has been used to treat cancer for several decades; it causes side effects when administered systemically which include myelosuppression and stomatitis which develop serious complications $(3,4)$. Therefore, to reduce this unwanted effect, the non-toxic prodrug converting $E$. coli $\mathrm{CD}$ system has recently received attention from researchers. The $\mathrm{CD}$ enzyme, translated from the $\mathrm{CD}$ gene converts non-toxic 5-FC into the cytotoxic 5-FU which inhibits cell growth selectively in the site where the gene is expressed (44). In our study, 5-FC-treated HB1.F3.CD cells increased in number when co-cultured with AGS indicating the use of this CD/5-FC GEPT system is possible after the injection of stem cells.

According to earlier reports, it was shown that a small number of CD-transfected cells can induce antitumor effects through a bystander effect (45), thus, we investigated whether the number of the GESTECs induce affected gastric cancer cells differently. When increasing number of the three stem cell lines $\left(8.0 \times 10^{3}, 1.6 \times 10^{4}\right.$ and $2.4 \times 10^{4}$ cells/well $)$ were cultured with AGS and equally treated with $5-\mathrm{FC}$ at $500 \mu \mathrm{g} / \mathrm{ml}, \mathrm{HB} 1$. F3.CD cells expressing the CD gene and HB1.F3.CD.IFN- $\beta$ cells expressing both the CD and IFN- $\beta$ fusion genes appeared to show maximum cancer cell growth inhibition starting at a 1:2 ratio of stem cells:AGS, results with higher stem cells number showed similar inhibition effects.

To examine if these gene expressing GESTECs are able to migrate to gastric cancer cells, we performed a modified transwell migration assay. Compared to bovine FB (i.e., control cells), the migration of cells increased in AGS, indicating that gastric cancer cells tend to secrete chemoattractant factors and GESTECs respond to them. In addition, this migrating capability of the parental HB1.F3 cells, was also shown in previous studies using melanoma, glioma, neuroblastoma prostate and breast tumors (11), indicating this cells line possesses a tendency to migrate towards variable types of cancer which can be an advantage for use as antitumor treatment.

Modified migration assay results made it possible to assume that gastric cancer cells might produce chemoattractant factors which induce the migration of HB1.F3.CD and HB1.F3.CD. IFN- $\beta$ cells to cancer cells, resulting in the delivery of therapeutic genes to the tumor site. Several factors such as SCF, VEGF are known to play a chemoattractive role in tumor cells $(10,12,14-27,46-49)$, but the details in gastric cancer cells are not clearly known. Thus, we assayed for chemoattractant ligands and receptors in AGS and found that SCF, CXCR4 and VEGF genes were expressed. Therefore, these genes may be related in tumor tropism of GESTECs that selectively deliver the suicide enzyme and anticancer cytokine genes to the gastric cancer site. Further study is required to confirm the role of these genes in the mechanism underlying tumor cell recognition and/or tumor tropism by GESTECs.

As explained previously, we studied whether the CD and IFN- $\beta$ fusion genes can maximize the antitumor effect. Since the mechanism of action of the two genes is different, a possible synergistic effect with the fusion gene was likely. $\mathrm{CD}$ acts as a pro-drug-activating enzyme (12) and IFN- $\beta$ can enhance anti-angiogenic effects and immune responses $(31,50)$. Results from this study showed that HB1.F3.CD.IFN- $\beta$ cells have significantly powerful antitumor effect compared to HB1.F3.CD cells. 
In conclusion, this study showed that the $\mathrm{CD}$ gene/5-FC modified GEPT system with the human IFN- $\beta$ GEPT system resulted in marked growth inhibition in gastric cancer cells. In addition, GESTECs expressing CD or CD with IFN- $\beta$ genes may selectively migrate toward gastric cancer cells. Therefore, it is possible to consider that GESTECs expressing suicide genes with an application of pro-drugs may have therapeutic potential for treating gastric cancer, and that GESTECs expressing the $\mathrm{CD}$ and IFN- $\beta$ fusion gene has a synergic antitumor effect compared to GESTECs expressing CD alone.

\section{Acknowledgements}

This study was supported by the National Research Foundation of Korea (NRF) grant (no. 2011-0015385) funded by the Korea government (MEST). In addition, this work was also supported by Priority Research Centers Program through the NRF funded by the Ministry of Education, Science and Technology (2009-0094035).

\section{References}

1. Isik M, Caner S, Metin Seker M, et al: Gastric adenocarcinoma under the age of 40; more metastatic, less differentiated. J BUON 16: 253-256, 2011

2. Blum M, Suzuki A and Ajani JA: A comprehensive review of S-1 in the treatment of advanced gastric adenocarcinoma. Future Oncol 7: 715-726, 2011.

3. Fidan E, Fidan S, Yildiz B, et al: Bolus fluorouracil induced syncope and pulseless ventricular tachycardia: a case report. Hippokratia 15: 93-95, 2011.

4. Longley DB, Harkin DP and Johnston PG: 5-Fluorouracil: mechanisms of action and clinical strategies. Nat Rev Cancer 3: 330-338, 2003

5. Luo XR, Li JS, Niu Y and Miao L: Targeted killing effects of double CD and TK suicide genes controlled by survivin promoter on gastric cancer cell. Mol Biol Rep 38: 1201-1207, 2011.

6. Anderson LM, Krotz S, Weitzman SA and Thimmapaya B: Breast cancer-specific expression of the Candida albicans cytosine deaminase gene using a transcriptional targeting approach. Cancer Gene Ther 7: 845-852, 2000.

7. Joo KM, Park IH, Shin JY, et al: Human neural stem cells can target and deliver therapeutic genes to breast cancer brain metastases. Mol Ther 17: 570-575, 2009.

8. Studeny M, Marini FC, Champlin RE, Zompetta C, Fidler IJ and Andreeff M: Bone marrow-derived mesenchymal stem cells as vehicles for interferon- $\beta$ delivery into tumors. Cancer Res 62 : 3603-3608, 2002.

9. Zhang JF, Wei F, Wang HP, et al: Potent anti-tumor activity of telomerase-dependent and HSV-TK armed oncolytic adenovirus for non-small cell lung cancer in vitro and in vivo. J Exp Clin Cancer Res 29: 52, 2010.

10. Aboody KS, Brown A, Rainov NG, et al: Neural stem cells display extensive tropism for pathology in adult brain: evidence from intracranial gliomas. Proc Natl Acad Sci USA 97: 12846-12851, 2000.

11. Aboody KS, Bush RA, Garcia E, et al: Development of a tumorselective approach to treat metastatic cancer. PLoS One 1: e23, 2006.

12. Kim SK, Kim SU, Park IH, et al: Human neural stem cells target experimental intracranial medulloblastoma and deliver a therapeutic gene leading to tumor regression. Clin Cancer Res 12: 5550-5556, 2006.

13. Kim KY, Kim SU, Leung PC, Jeung EB and Choi KC: Influence of the prodrugs 5-fluorocytosine and CPT-11 on ovarian cancer cells using genetically engineered stem cells: tumor-tropic potential and inhibition of ovarian cancer cell growth. Cancer Sci 101: 955-962, 2010

14. Kim SU: Human neural stem cells genetically modified for brain repair in neurological disorders. Neuropathology 24: 159-171, 2004
15. Kim SU, Nakagawa E, Hatori K, Nagai A, Lee MA and Bang JH: Production of immortalized human neural crest stem cells. Methods Mol Biol 198: 55-65, 2002.

16. Evoy D, Hirschowitz EA, Naama HA, et al: In vivo adenoviralmediated gene transfer in the treatment of pancreatic cancer. J Surg Res 69: 226-231, 1997.

17. Hirschowitz EA, Ohwada A,Pascal WR, Russi TJ and Crystal RG In vivo adenovirus-mediated gene transfer of the Escherichia coli cytosine deaminase gene to human colon carcinoma-derived tumors induces chemosensitivity to 5-fluorocytosine. Hum Gene Ther 6: 1055-1063, 1995.

18. Kanai F, Lan KH, Shiratori $\mathrm{Y}$, et al: In vivo gene therapy for $\alpha$-fetoprotein-producing hepatocellular carcinoma by adenovirus-mediated transfer of cytosine deaminase gene. Cancer Res 57: 461-465, 1997.

19. Lan KH, Kanai F, Shiratori Y, et al: Tumor-specific gene expression in carcinoembryonic antigen - producing gastric cancer cells using adenovirus vectors. Gastroenterology 111: 1241-1251, 1996.

20. Li Z, Shanmugam N, Katayose D, et al: Enzyme/prodrug gene therapy approach for breast cancer using a recombinant adenovirus expressing Escherichia coli cytosine deaminase. Cancer Gene Ther 4: 113-117, 1997.

21. Austin EA and Huber BE: A first step in the development of gene therapy for colorectal carcinoma: cloning, sequencing, and expression of Escherichia coli cytosine deaminase. Mol Pharmacol 43: 380-387, 1993.

22. Mullen CA, Kilstrup M and Blaese RM: Transfer of the bacterial gene for cytosine deaminase to mammalian cells confers lethal sensitivity to 5-fluorocy tosine: a negative selection system. Proc Natl Acad Sci USA 89: 33-37, 1992.

23. Etienne MC, Cheradame S, Fischel JL, et al: Response to fluorouracil therapy in cancer patients: the role of tumoral dihydropyrimidine dehydrogenase activity. J Clin Oncol 13: $1663-1670,1995$

24. Pinedo HM and Peters GF: Fluorouracil: biochemistry and pharmacology. J Clin Oncol 6: 1653-1664, 1988.

25. Chung-Faye GA, Chen MJ, Green NK, et al: In vivo gene therapy for colon cancer using adenovirus-mediated, transfer of the fusion gene cytosine deaminase and uracil phosphoribosyltransferase. Gene Ther 8: 1547-1554, 2001

26. Crystal RG, Hirschowitz E, Lieberman M, et al: Phase I study of direct administration of a replication deficient adenovirus vector containing the $E$. coli cytosine deaminase gene to metastatic colon carcinoma of the liver in association with the oral administration of the pro-drug 5-fluorocytosine. Hum Gene Ther 8: 985-1001, 1997.

27. Freytag SO, Khil M, Stricker H, et al: Phase I study of replicationcompetent adenovirus-mediated double suicide gene therapy for the treatment of locally recurrent prostate cancer. Cancer Res 62 : 4968-4976, 2002.

28. Dong Z, Greene G, Pettaway C, et al: Suppression of angiogenesis, tumorigenicity, and metastasis by human prostate cancer cells engineered to produce interferon- $\beta$. Cancer Res 59: 872-879, 1999 .

29. Rossiello F, De Cicco Nardone F and Dell'Acqua S: Interferon- $\beta$ increases the sensitivity of endometrial cancer cells to cellmediated cytotoxicity. Gynecol Oncol 54: 130-136, 1994.

30. Yi BR, Hwang KA, Kang NH, Kim SU, Jeung EB and Choi KC: Antitumor therapeutic effects of cytosine deaminase and interferon- $\beta$ against endometrial cancer cells using genetically engineered stem cells in vitro. Anticancer Res 31: 2853-2862, 2011.

31. Yi BR, O SN, Kang NH, et al: Genetically engineered stem cells expressing cytosine deaminase and interferon- $\beta$ migrate to human lung cancer cells and have potentially therapeutic antitumor effects. Int J Oncol 39: 833-839, 2011.

32. Schmidt NO, Przylecki W, Yang W, et al: Brain tumor tropism of transplanted human neural stem cells is induced by vascular endothelial growth factor. Neoplasia 7: 623-629, 2005.

33. Sun L, Lee J and Fine HA: Neuronally expressed stem cell factor induces neural stem cell migration to areas of brain injury. J Clin Invest 113: 1364-1374, 2004.

34. Ehtesham M, Yuan X, Kabos P, et al: Glioma tropic neural stem cells consist of astrocytic precursors and their migratory capacity is mediated by CXCR4. Neoplasia 6: 287-293, 2004.

35. Jeong SW, Chu K, Jung KH, Kim SU, Kim M and Roh JK: Human neural stem cell transplantation promotes functional recovery in rats with experimental intracerebral hemorrhage. Stroke 34: 2258-2263, 2003. 
36. Kim SU, Park IH, Kim TH, et al: Brain transplantation of human neural stem cells transduced with tyrosine hydroxylase and GTP cyclohydrolase 1 provides functional improvement in animal models of Parkinson disease. Neuropathology 26: 129-140, 2006.

37. Meng XL, Shen JS, Ohashi T, Maeda H, Kim SU and Eto Y: Brain transplantation of genetically engineered human neural stem cells globally corrects brain lesions in the mucopolysaccharidosis type VII mouse. J Neurosci Res 74: 266-277, 2003.

38. Rosser AE, Zietlow R and Dunnett SB: Stem cell transplantation for neurodegenerative diseases. Curr Opin Neurol 20: 688-692, 2007.

39. Ryu JK, Kim J, Cho SJ, et al: Proactive transplantation of human neural stem cells prevents degeneration of striatal neurons in a rat model of Huntington disease. Neurobiol Dis 16: 68-77, 2004.

40. Lee ST, Chu K, Park JE, et al: Intravenous administration of human neural stem cells induces functional recovery in Huntington's disease rat model. Neurosci Res 52: 243-249, 2005.

41. Lee DH, Ahn Y, Kim SU, et al: Targeting rat brainstem glioma using human neural stem cells and human mesenchymal stem cells. Clin Cancer Res 15: 4925-4934, 2009.

42. Boucher PD, Im MM, Freytag SO and Shewach DS: A novel mechanism of synergistic cytotoxicity with 5-fluorocytosine and ganciclovir in double suicide gene therapy. Cancer Res 66: 3230-3237, 2006.

43. Hartmann KU and Heidelberger C: Studies on fluorinated pyrimidines. XIII. Inhibition of thymidylate synthetase. J Biol Chem 236: 3006-3013, 1961.
44. Wei J, Wahl J, Knauss H, et al: Cytosine deaminase/5-fluorocytosine gene therapy and Apo2L/TRAIL cooperate to kill TRAIL-resistant tumor cells. Cancer Gene Ther 14: 640-651, 2007.

45. Huber BE, Austin EA, Richards CA, Davis ST and Good SS: Metabolism of 5-fluorocytosine to 5-fluorouracil in human colorectal tumor cells transduced with the cytosine deaminase gene: significant antitumor effects when only a small percentage of tumor cells express cytosine deaminase. Proc Natl Acad Sci USA 91: 8302-8306, 1994.

46. Saukkonen K and Hemminki A: Tissue-specific promoters for cancer gene therapy. Expert Opin Biol Ther 4: 683-696, 2004.

47. Tubiana M: Tumor cell proliferation kinetics and tumor growth rate. Acta Oncol 28: 113-121, 1989.

48. Beppu K, Jaboine J, Merchant MS, Mackall CL and Thiele CJ: Effect of imatinib mesylate on neuroblastoma tumorigenesis and vascular endothelial growth factor expression. J Natl Cancer Inst 96: 46-55, 2004.

49. Sun L, Hui AM, Su Q, et al: Neuronal and glioma-derived stem cell factor induces angiogenesis within the brain. Cancer Cell 9: 287-300, 2006

50. Nakamizo A, Marini F, Amano T, et al: Human bone marrowderived mesenchymal stem cells in the treatment of gliomas. Cancer Res 65: 3307-3318, 2005. 